\title{
Research on a New Control Scheme of Photovoltaic Grid Power Generation System
}

\author{
Dong-Hui Li, Yi-Lin Wang, and Da-jun Zhou \\ School of Electrical Engineering and Automation, Tianjin University, Tianjin 300072, China \\ Correspondence should be addressed to Dong-Hui Li; lidonghui@tju.edu.cn
}

Received 24 January 2014; Accepted 10 July 2014; Published 10 August 2014

Academic Editor: Gongnan Xie

Copyright ( 2014 Dong-Hui Li et al. This is an open access article distributed under the Creative Commons Attribution License, which permits unrestricted use, distribution, and reproduction in any medium, provided the original work is properly cited.

\begin{abstract}
A new type of photovoltaic grid power generation system control scheme to solve the problems of the conventional photovoltaic grid power generation systems is presented. To aim at the oscillation and misjudgment of traditional perturbation observation method, an improved perturbation observation method comparing to the next moment power is proposed, combining with BOOST step-up circuit to realize the maximum power tracking. To counter the harmonic pollution problem in photovoltaic grid power generation system, the deadbeat control scheme in fundamental wave synchronous frequency rotating coordinate system of power grid is presented. A parameter optimization scheme based on positive feedback of active frequency shift island detection to solve the problems like the nondetection zone due to the import of disturbance in traditional island detection method is proposed. Finally, the results in simulation environment by MATLAB/Simulink simulation and experiment environment verify the validity and superiority of the proposed scheme.
\end{abstract}

\section{Introduction}

Solar energy has no noise and no pollution and is sustainable with a series of advantages. It has become one of the most potential green energies for development. Photovoltaic power generation is one of the main forms of solar energy utilization. In-depth study of photovoltaic power generation technology for solving the energy crisis can reduce environmental pollution and promote healthy economic development with great strategic significance.

Grid-connected PV systems still have many technical problems to be solved. Firstly, since the output power of the solar cell is easily influenced by environmental factors with obvious nonlinear factor, the photovoltaic cell is a very unstable power supply, so designing an efficient and reliable maximum power tracking control strategy and output as much energy in different light and temperature conditions to improve the efficiency of the system is an important issue. Secondly, since the inverter is running excessive harmonic currents injected into the grid, the power system harmonic pollution problem is getting worse. Grid system becomes abnormal and false, and another equipment is also adversely affected. Thirdly, simple instructions had no effect on current perturbation scheme, so it is needed to have more reliable and stable detection methods to prevent islanding effect. How to more effectively reduce the impact of islanding detection algorithm on the output power disturbances and reduce nondetection spots are the focuses of islanding detection research.

To solve the above problems, this paper proposes a new grid-connected PV system solution. The program uses boost circuit combined with improved perturbation and observation method for maximum power point tracking in order to reduce the volatility of photovoltaic power generation and further reduce the harmonic content of the network current. The paper proposes deadbeat control strategy in the fundamental frequency power synchronous rotating coordinate in order to adapt to the grid connected and little influence on power quality. The network can quickly detect the islanding effect. This paper proposes the active frequency drift islanding detection method with positive feedback on the basis of islanding detection method. 


\section{Maximum Power Point Tracking}

2.1. Improved Perturbation and Observation Method. Although the traditional perturbation and observation method is easy to be implemented, the step size selection and environmental factors cause an "oscillation" and "misjudgment" serious phenomenon. They will reduce the maximum tracking speed and accuracy [1]. Based on the above problems, the paper presents an improved perturbation and observation method increasing the link compared with the next moment power. That is to say, the system achieves the maximum power point tracking by judging the disturbing slope and comparing with the next time power. This method can quickly track near the maximum power point and can also correct the tracking if sudden changes happen in external conditions. It not only does not cause tracking failure or error but also effectively improves the tracking precision and reduces the power loss. Figure 1 is a flowchart of improved perturbation and observation method.

2.2. The Maximum Power Point Tracking Control. Since conversion efficiency of PV power generation system is low, the important thing is to adjust the working point of photovoltaic array and keep it working near the maximum power point to improve the overall efficiency of the system power. But in the solar cell power supply system, the internal resistance of solar cell is affected not only by the intensity of sunlight but also by the ambient temperature. So the solar cell is always unstable and it is impossible to gain the maximum power output by the above-described simple methods. The current method to achieve the maximum power point tracking control is to add a DC/DC converter between the solar array and the load, which adjusts the solar array in the maximum power point by changing the duty cycle of power DC/DC converter switch.

This paper tracks the maximum power point of solar panels by utilizing the method of the boost circuit with the improved perturbation and observation.

Figure 2 shows that the solar cell will detect the output voltage and output current signal and put it into the MPPT algorithm module, and the power is obtained by multiplying the output of the solar cell. Then by the improved perturbation and observation method, it will output a modulated signal after calculation. Comparing the modulated signal with the triangular wave signal with constant frequency, it generates a switching signal, which can control the power switching device of the booster circuit. The system achieves the purposes of maximum power point tracking by adjusting the duty ratio of the power switching devices.

\section{Grid Control Strategy}

Photovoltaic grid-connected system needs to use the gridconnected inverter as power conversion device of the DC output of the PV array into AC power. The power grid has strict requirements on the current harmonic content by inverter, and it is particularly important in the grid control strategy to control the current output waveform of the inverter $[2,3]$.

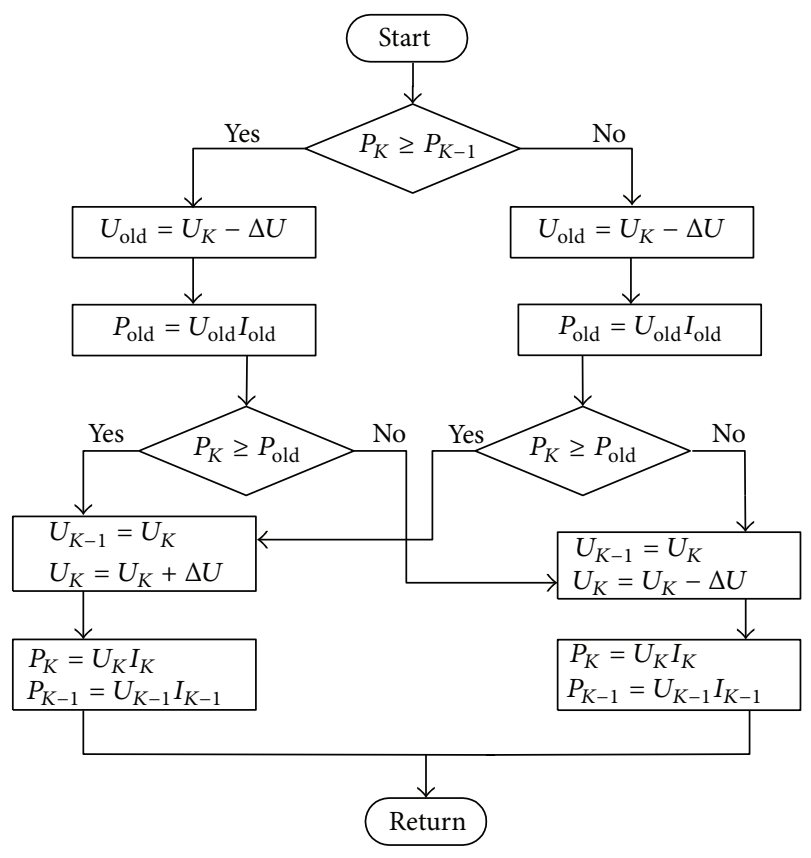

FIgURE 1: Flowchart of improved perturbation and observation method.

The inverter usually controls the output current of the inverter by a pulse width modulation control technology. There are many ways to achieve the control of the current inner loop by current-controlled voltage source inverter. The hysteresis current control is dynamic and has fast response in the literature [4], but the hysteresis loop width is fixed. So the switching frequency of the power devices is not fixed, and the reliability of the circuit is decreased. In the literature [5], it prevents PI control in the synchronous rotating coordinate system, which puts the voltage and current components into DC component, which can achieve floating control. But by a series of tests, the parameters of PI controller are set, and better performance parameters can be obtained. The deadbeat control with the current deadbeat PWM algorithm has a good dynamic response, and the output current can quickly and accurately track the reference current [6]. In recent years, the deadbeat control is combined with space vector pulse width modulation, which is widely used in the power electronic converters with digital control $[7,8]$. This paper establishes the model of the three-phase grid-connected inverter discrete mathematical with deadbeat control based on the SVPWM current and proposes the deadbeat control strategy in the fundamental frequency power synchronous rotating coordinate.

3.1. In the $d-q$ Coordinate System Deadbeat Current Control. Topology of two-stage three-phase PV inverter is shown in Figure 3. In the figure, physical quantities are defined as follows: $U_{a}, U_{b}$, and $U_{c}$ are the inverter output voltages; $E_{a}, E_{b}$, and $E_{c}$ are three-phase photovoltaic grid system grid voltages; $I_{a}, I_{b}$, and $I_{c}$ are three-phase grid-connected system output inductor currents; $L$ is the filter inductor; $L_{1}$ is the boost inductor; and $R$ is circuit equivalent resistance. 


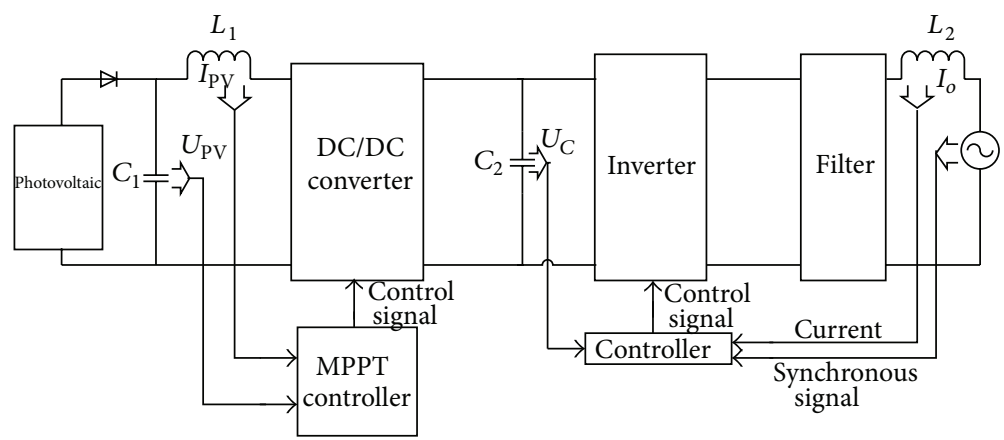

FIGURE 2: Schematic diagram of maximum power point tracking using boost circuit.

As shown in Figure 3, in the three-phase static $a, b, c$ coordinate system, according to Kirchhoff's law, the circuit equations of the model are listed as follows:

$$
\begin{aligned}
& U_{a}=L \frac{d I_{a}}{d t}+I_{a} \cdot R+E_{a} \\
& U_{b}=L \frac{d I_{b}}{d t}+I_{b} \cdot R+E_{b} \\
& U_{c}=L \frac{d I_{c}}{d t}+I_{c} \cdot R+E_{c} .
\end{aligned}
$$

$3 / 2$ conversion of (1) and rotation can be obtained by the $d-q$ coordinate model of grid voltage and frequency synchronization rotation:

$$
\begin{aligned}
& U_{d}=L \frac{d I_{d}}{d t}+I_{d} \cdot R-\omega L \cdot I_{q}+E_{d}, \\
& U_{q}=L \frac{d I_{q}}{d t}+I_{q} \cdot R-\omega L \cdot I_{d}+E_{q} .
\end{aligned}
$$

In the equations, $U_{d}$ and $U_{q}$ are the vector of the output voltage of the inverter $d, q$ components. $E_{d}$ and $E_{q}$ are the three-phase grid voltage vector $d, q$ components. $I_{d}$ and $I_{q}$ are the inverter output current vector $d, q$ components, and $\omega$ is the grid voltage and frequency.

Equation (2) of state is obtained, and the control cycle is set to T. A discrete state equation of the three-phase PV inverters discrete model is

$$
\begin{aligned}
{\left[\begin{array}{c}
I_{d}(k+1) \\
I_{q}(k+1)
\end{array}\right]=} & {\left[\begin{array}{cc}
\frac{L-R T}{L} & -\omega \\
\frac{L-R T}{L} & \omega T
\end{array}\right]\left[\begin{array}{c}
I_{d}(k) \\
I_{q}(k)
\end{array}\right] } \\
& +\left[\begin{array}{cccc}
\frac{T}{L} & 0 & -\frac{T}{L} & 0 \\
0 & \frac{T}{L} & 0 & -\frac{T}{L}
\end{array}\right]\left[\begin{array}{c}
U_{d}(k) \\
U_{q}(k) \\
E_{d}(k) \\
E_{q}(k)
\end{array}\right] .
\end{aligned}
$$

By (4) the expression of deadbeat control voltage directive can be obtained:

$$
\begin{aligned}
U_{d}(k)= & \frac{L}{T} \cdot I_{d}(k+1)-\frac{L-R \cdot T}{T} \cdot I_{d}(k) \\
& -\omega L \cdot I_{q}(k)-E_{d}(k), \\
U_{q}(k)= & \frac{L}{T} \cdot I_{q}(k+1)-\frac{L-R \cdot T}{T} \cdot I_{q}(k) \\
& -\omega L \cdot I_{d}(k)-E_{q}(k) .
\end{aligned}
$$

By formula (4) it obtains $U_{d}(k)$ and $U_{d}(k)$, and after converting through $2 r / 2 s$ the control signal of SVPWM inverter switching devices is generated.

3.2. The Overall Control Strategy of Grid-Connected Inverter. The structure of three-phase PV inverter control is shown in Figure 4. The entire system is composed of a maximum power tracking MPPT controller, DC voltage, and AC current PI controller deadbeat controller. The control system uses the double-loop control structure of voltage outer loop and current inner loop. The voltage outer loop can keep DC voltage stable, and the current inner loop is used to improve the rapidity of the system.

\section{Islanding Detection Method and Parameter Optimization}

4.1. Auto Frequency Drift Method. The islanding effect of the grid-connected PV systems does great harm to the maintenance personnel as well as the equipment of the power grid. Therefore, anti-islanding strategy must be added to the grid-connected PV systems [8]. Auto frequency drift (AFD) method has a high possibility to detect the islanding effect and there is no need to add any hardware to the system. However, the parameter and the algorithm can greatly affect the capacity of AFD. If the parameter is set small and even though the disturbance to the power grid is small, it is possible to leak the detection of islanding effect. If the parameter is set large, the islanding effect can be detected easily, but it can make the quality of the power poor even it may cause the voltage changes and make the power system unstable $[9,10]$. Besides, when the circuit presents capacitive, 


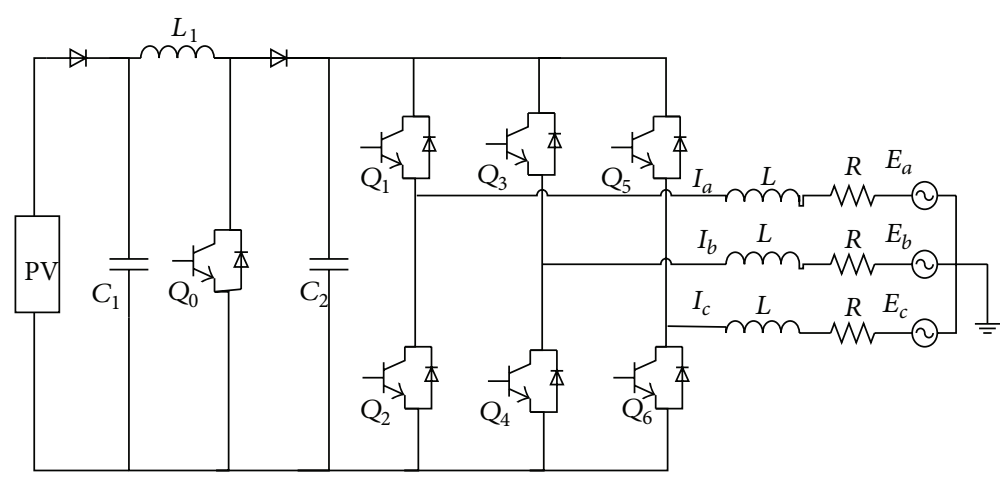

FIGURE 3: Structure diagram of three-phase photovoltaic grid-connected inverter.

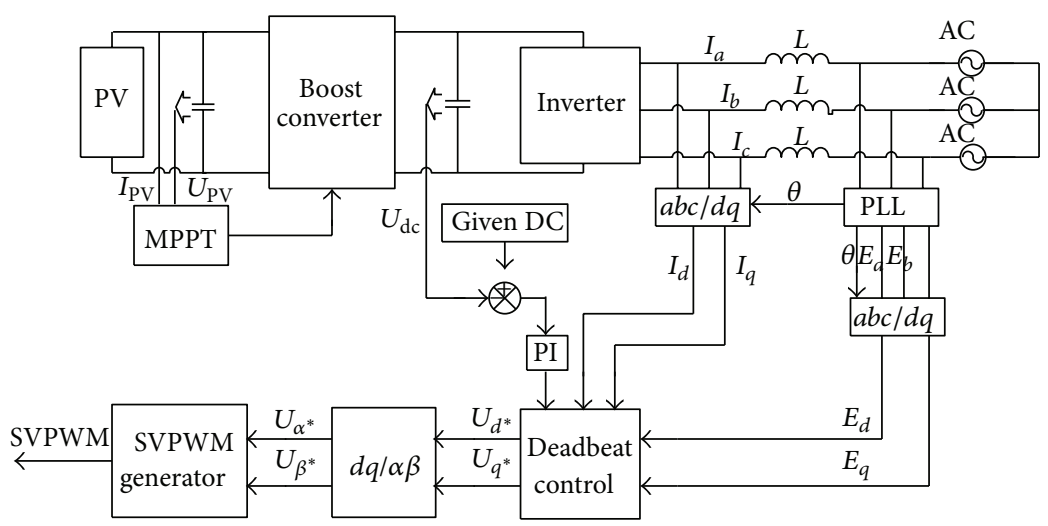

FIGURE 4: Structure of three-phase photovoltaic grid-connected inverter.

the decline of the frequency can help detect the islanding effect, and the increase of the frequency may cause the failure to detect the islanding effect because of the offset of the load phase angle. When the circuits presents inductive, the increase of the frequency can help detect the islanding effect, and the decline of the frequency can create the nondetection zone. So that, according to the character of AFD algorithm, in order to have little influence on the quality of power, to detect the islanding effect quickly when off the grid, to make the nondetection zone small, or to make nondetection zone under some specific loads, the paper introduces the positive feedback to AFD algorithm, which is active frequency with positive feedback (AFDPF).

For linear AFDPF, its control strategy is

$$
c_{f}=c_{f 0}+k \Delta f
$$

In the formula, $c_{f}$ is truncation functions; $c_{f 0}$ is fixed initial perturbation; $\Delta f=f-f_{0}$ is the difference between the common point frequency and the grid frequency; $k$ is the feedback gain.

4.2. Parameter Optimization of AFDPF Algorithm. When the grid voltage decreases, if the inverter does not separate from the power grid, the common point frequency fluctuations will occur until a new equilibrium is generated. $Q_{f 0}$ and $C_{\text {nom }}$ are parameters defined in (6) to (8):

$$
\begin{gathered}
Q_{f 0}=\omega_{0} R C=\frac{R}{\omega_{0} R}, \\
C_{0}=\frac{1}{\omega_{0}^{2} L}, \\
C_{\text {nom }}=\frac{C}{C_{0}} .
\end{gathered}
$$

$Q_{f 0}$ is an inherent characteristic of the RLC circuit, which is decided by the $R, L$, and $C ; Q_{f 0}$ is determined by the grid frequency $\omega_{0}, R$, and $L$; however, $C$ is irrelevant from $Q_{f 0}$. When the active power is matching, $\omega_{0}$ and $R$ are constant, so the $Q_{f 0}$ only is determined by $L$.

The relationship between load capacitor and resonant capacitor is

$$
C=C_{\text {nom }} C_{0}=(1+\Delta C) C_{0}
$$

Angular frequency of the common coupling point is

$$
\omega=\omega_{0}+\Delta \omega
$$

In the equation, $\omega_{0}$ is the angular frequency of the grid, and $\omega_{0}=2 \pi f_{0}=1 / \sqrt{C L}$. 


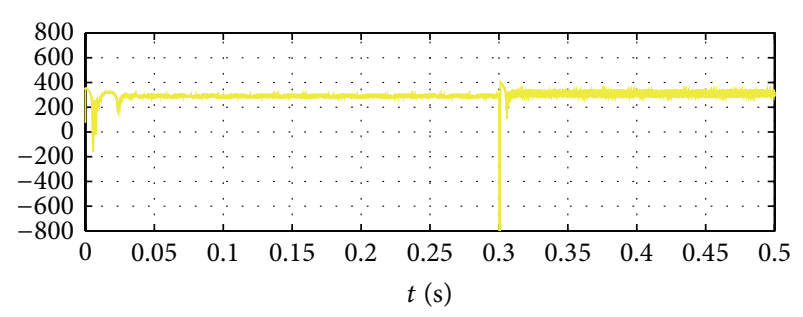

(a)

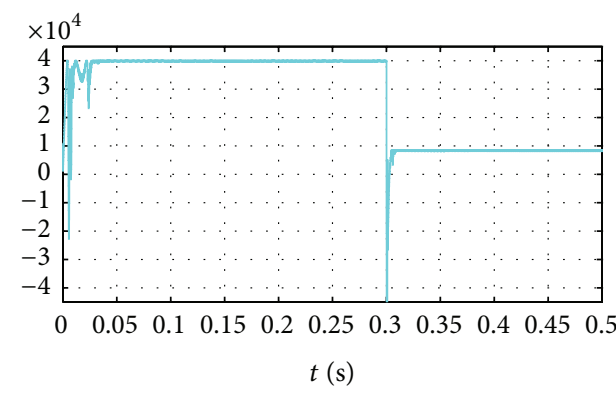

(c)

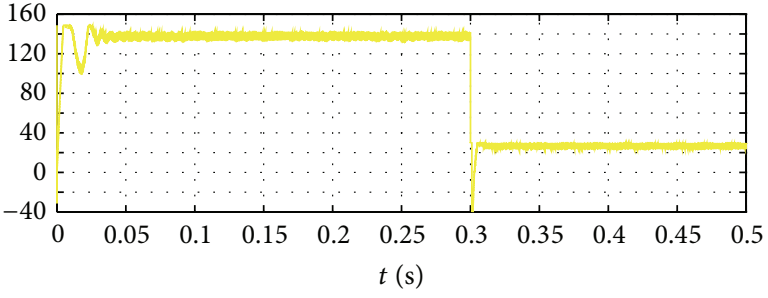

(b)

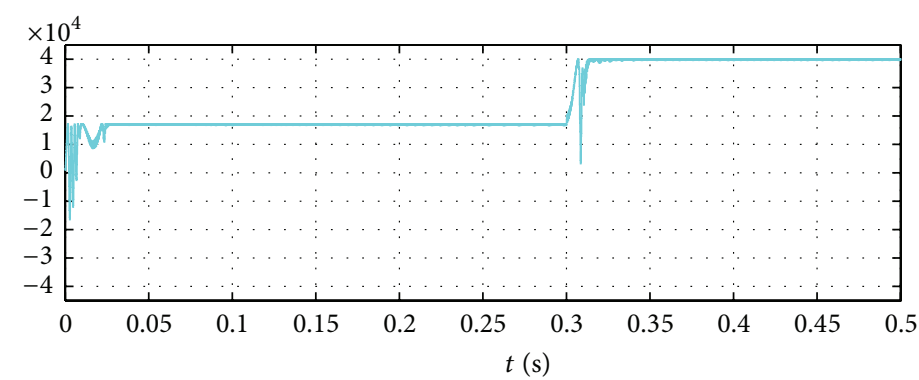

(d)

FIGURE 5: Maximum power point tracking simulation waveforms.

AFDPF method corresponds to the criterion:

$$
\arctan \left[R\left(\frac{1}{\omega L}-\omega C\right)\right]=\frac{\omega t_{z}}{2}=\frac{\pi c_{f}}{2} .
$$

In the equation, $t_{z}$ is the time interval when current crosses zero leading (or lagging) the voltage to cross zero.

Substituting (6) to (9) into (11) gets

$$
C_{\text {nom }}=\frac{\tan \left(\pi c_{f 0} / 2+\pi k \Delta f / 2\right)}{Q_{f 0}}-\frac{2 \Delta \omega}{\omega_{0}}+1 .
$$

The frequency difference $\Delta f$ between the common point and the power grid ranges from -0.7 to $0.5 \mathrm{~Hz}$, and fixed initial perturbation can be assumed $c_{f 0}=0.02$.

In order to make the detection having no nondetection zone, the boundary of the capacitance value should be

$$
C_{\text {nom }}^{+}-C_{\text {nom }}^{-}<0 \text {. }
$$

So it gets

$$
k>\frac{8 Q_{f 0}}{\omega_{0}} .
$$

When $f_{0}=50 \mathrm{~Hz}$, it can get the following result: $k>$ 0.0637 .

Thus, in order to make the nondetection zone smallest, feedback gain value should be at least 0.0637 .

According to the theory above, the paper deduces the range of the feedback gain value of the AFDPF nondetection zone under some specific load, which provides the reference of the parameter optimizations for the islanding detection method.

\section{Simulation}

In this paper, in the MATLAB/Simulink simulation environment, the maximum power point tracking, deadbeat control strategy, and islanding detection simulation models are established. The paper shows the simulation results.

5.1. Maximum Power Point Tracking. According to the MPPT control strategy, simulation model is built. When the light intensity reduces, the waveforms of the maximum power point voltage and current are shown in Figures 5(a) and $5(\mathrm{~b})$. Figures 5(c) and 5(d) are a variation of light intensity and temperature, the output power of the solar panel variation waveform. The result can get the fact that when the radiation intensity changes, the improved disturbance observer method can respond rapidly to achieve maximum power point tracking of the photovoltaic. Under different light intensities, in order to track the maximum power point, the duty cycle of the pulse control signal of the MPPT controller output is different, which verifies the theoretical analysis of the PV maximum power point tracking.

5.2. Deadbeat Control Strategy. This paper verifies the correctness of the deadbeat control in the $d$ - $q$ coordinate system by MATLAB/Simulink simulation. Simulation parameters are as follows: grid voltage is $220 \mathrm{~V}$, frequency is $50 \mathrm{~Hz}$, three-phase inverter AC measuring inductance is $3 \mathrm{mH}, \mathrm{AC}$ resistance measurement is $0.1 \Omega$, simulation time is $0.5 \mathrm{~s}$, and the switching frequency is $10 \mathrm{KHz}$.

When at $0.3 \mathrm{~s}$, the light intensity drops from $1000 \mathrm{~W} / \mathrm{m}^{2}$ to $400 \mathrm{~W} / \mathrm{m}^{2}$, and the DC voltage causes slight fluctuations at this time; the output current waveform of inverter is declining with light intensity decreasing, which is the same with the grid voltage waveform, and they are the same frequency 


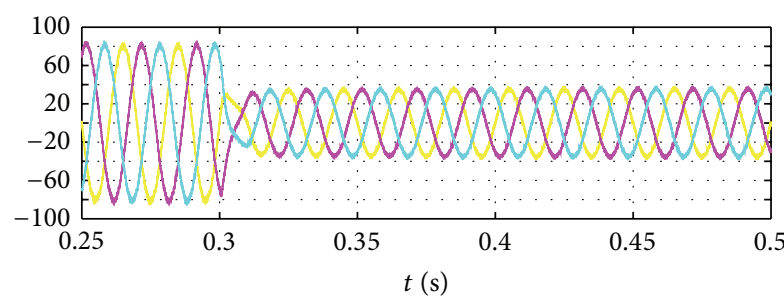

(a) Three-phase grid current

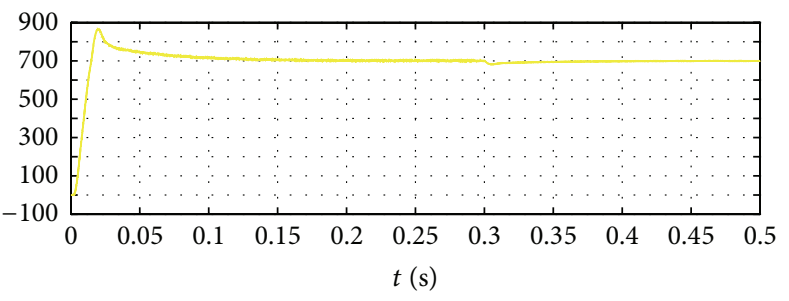

(c) PV inverter DC bus voltage

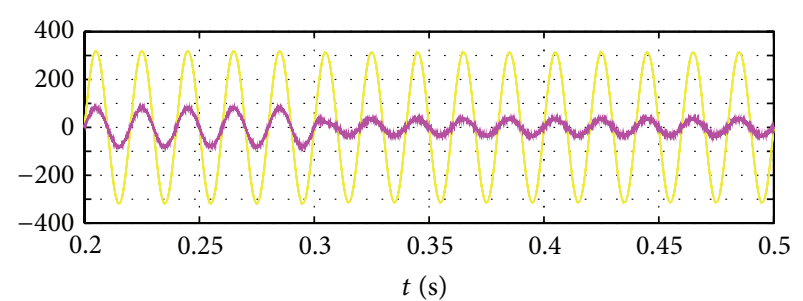

(b) Single-phase current and voltage grid

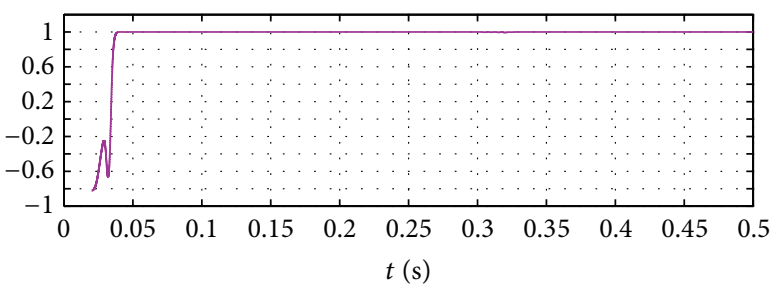

(d) Grid power factor

FIGURE 6: The results of output three-phase grid-connected inverter simulation.
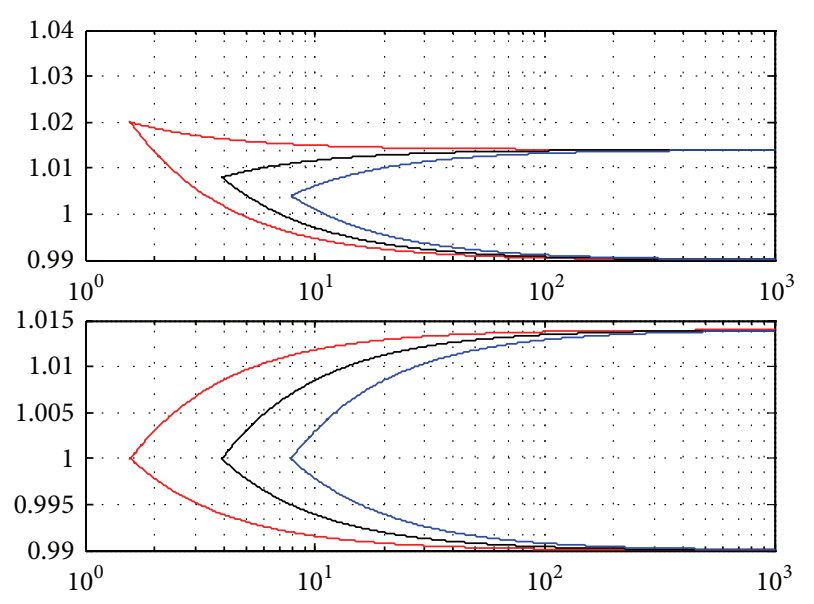

FIgURE 7: The nondetection zone of AFDPF.

and phase, and the power factor is 1, as shown in Figure 6. When the light intensity is changed, the current waveform of inverter output can be stabilized in less than one cycle time, and the DC bus voltage will remain stable. The waveform of output current of the inverter is a sine wave, and it is the same with the grid voltage with frequency and phase waveform and achieves the power to feed the grid with unit power factor.

From the MATLAB/Simulation results, the control strategy has good dynamic and steady-state performance and reduces the harmonic content of the current network. The inverter output voltage waveform is a sine wave with the same frequency and phase power, and the control strategies achieve the delivery by unity power factor into the grid.

5.3. Islanding Detection. Through MATLAB simulation, the distribution of the nondetection zone of AFDPF is shown in Figure 7.
In the top of Figure 7, the left curve is prescribed $c_{f}=0.02$ $+0.02 \Delta f$, the middle one is prescribed $c_{f}=0.02+0.05 \Delta f$, and the right one is prescribed $c_{f}=0.02+0.1 \Delta f$.

In the bottom of Figure 7, the left curve is prescribed $c_{f}=$ $0.02 \Delta f$, the middle one is $c_{f}=0.05 \Delta f$, and the right one is prescribed $c_{f}=0.1 \Delta f$.

The size of the nondetection zone is not affected by the truncated coefficient $c_{f}$ of the AFDPF algorithm for a given load value $Q_{f}$. But the larger $c_{f 0}$ is, the higher the nondetection zone is. So AFDPF algorithm by increasing the scaling factor $k$ reduces the nondetection zone. If there is no nondetection zone until it reaches a given value $Q_{f}$.

Figure 8 shows that $k$ parameters of AFDPF improve the reliability of islanding detection and reduce nondetection zone.

\section{Experiment Verification}

In order to prove the whole control scheme, this paper uses TOPCON $10 \mathrm{~kW}$ photovoltaic array simulator from Regatron Corporation to do the experiment. In this experiment, we design a two-stage inverter; in the first stage, we use boost circuit to elevate the DC voltage; in the second stage, we use IGBT full-bridge inverter to connect to the power grid. The DSP of the inverter we use TMS320F2812 from TI. The value of the filter inductance in AC side is $12 \mathrm{mH}$.

In this paper, according to Figure 4 , the whole control scheme, we finish the experiment. The DC voltage wave of the inverter in the first stage is shown in Figure 9; the output current wave of the inverter and the voltage wave of the power grid are shown in Figure 10 (the voltage wave has a larger amplitude). Besides we measure the power factor equals 1 , the current's THD equals $2.2 \%$. Therefore, the DC voltage has low fluctuation and ripple wave, and the power factor equals 1 when the current merges into the power grid. 


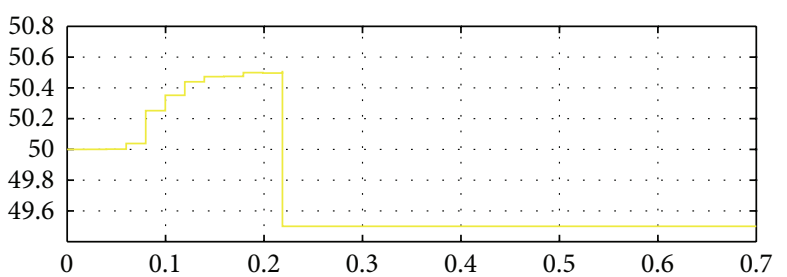

(a)

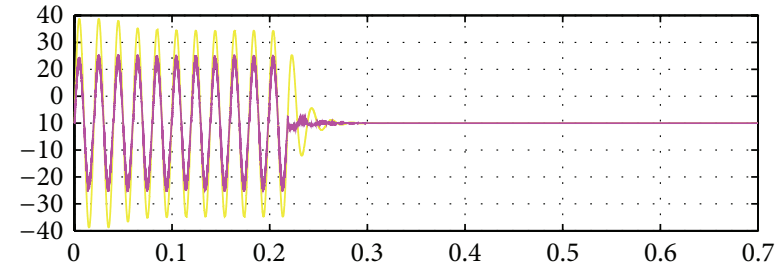

(b)

FIGURE 8: AFDPF the simulation waveforms.

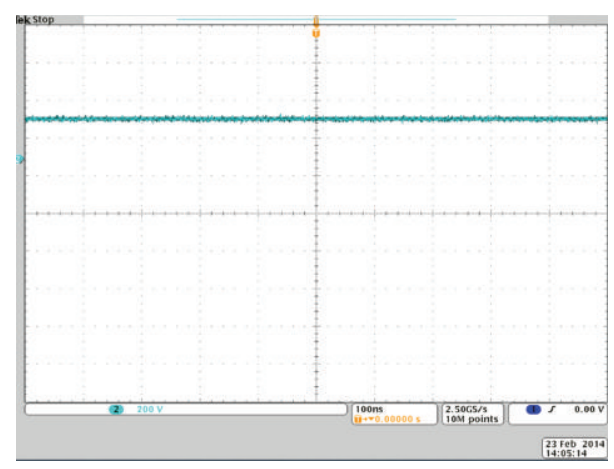

FIGURE 9: DC voltage wave.

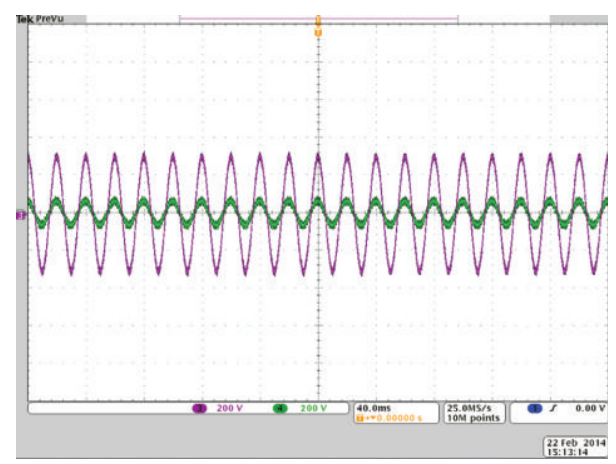

FIgURE 10: The inverter current wave and the power grid voltage wave.

\section{Conclusion}

To aim at conventional problems in photovoltaic grid power generation system, a new scheme of photovoltaic grid power generation system is proposed. According to the MAT$\mathrm{LAB} /$ Simulink simulation as well as the experiment, it is verified that the strategy can ameliorate the phenomenon of oscillation and misjudgment in the conventional disturbance observation method. Faster and more reliable maximum power tracking of photovoltaic cell can also be achieved. The scheme uses the deadbeat control of the three-phase photovoltaic grid inverter in $d$-q coordinates and effectively reduces the content of harmonic wave of the output current in photovoltaic power generation system. The power delivery to the grid by unit power factor is achieved. The active frequency drift with positive feedback method further reduces the nondetection zone by introducing the parameter values of $k$ and improves the reliability of island detection.

\section{Conflict of Interests}

The authors declare that they have no financial or personal relationships with other people or organizations that can inappropriately influence their work. Besides, there is no official or other personal conflict of interests in this paper.

\section{References}

[1] V. Salas, E. Olias, A. Barrado, and A. Lfizaro, "Review of the maximum power point tracking algorithms for stand-alone photovoltaic systems," Solar Energy Materials and Solar Cells, vol. 90, no. 11, pp. 1555-1578, 2006.

[2] L. Hassaine, E. Olias, J. Quintero, and M. Haddadi, "Digital power factor control and reactive power regulation for gridconnected photovoltaic inverter," Renewable Energy, vol. 34, no. 1, pp. 315-321, 2009.

[3] M. Hagiwara, K. Wada, H. Fujita, and H. Akagi, "Dynamic behavior of a 21-level BTB-based power-flow controller under single-line-to-ground fault conditions," IEEE Transactions on Industry Applications, vol. 43, no. 5, pp. 1379-1387, 2007.

[4] G. He-rong, Y. Zi-long, and W. Wu, "Research on hysteresisband current tracking control of grid-connected inverter," Proceedings of the Chinese Society of Electrical Engineering, vol. 26, no. 9, pp. 108-112, 2006.

[5] W.-F. Zhu, W. Dou, and Z.-G. Xu, "Design of the PI current controller of three phase photovoltaic grid-connected inverter," Renewable Energy Resources, vol. 27, no. 2, pp. 55-58, 2009.

[6] T. Huang, X. Shi, D. Wei, Y. Sun, and D. Wang, "Study on threephase photovoltaic grid-connected inverter based on current deadbeat control," Power System Protection and Control, vol. 40, no. 11, pp. 36-40, 2012.

[7] Q. Rui, S. Du, and D. Jiang, "Current regulation for three-phased grid-connected inverter," Power Electronics, vol. 44, no. 4, pp. 45, 2010.

[8] F. Liu, Y. Kang, S. Duan, Z. Wang, and H. Wang, "Parameter optimization of active frequency drift islanding detection method," Proceedings of the Chinese Society of Electrical Engineering, vol. 28, no. 1, pp. 95-99, 2008.

[9] S.-C. Zheng, M. Ding, J.-H. Su, M. Mao, and G. Zhang, "Simulation and experiment research of photovoltaic generation system and its islanding," Journal of System Simulation, vol. 17, no. 12, pp. 3085-3088, 2005.

[10] K. Senlki, J. Jinhong, C. Changhee et al., "Modeling and simulation of grid-connected PV generation system for electromag netic transient analysis," Solar Energy, vol. 10, no. 8, pp. 1-15, 2011. 


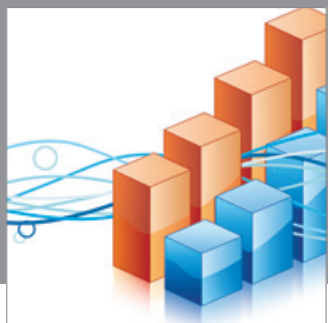

Advances in

Operations Research

mansans

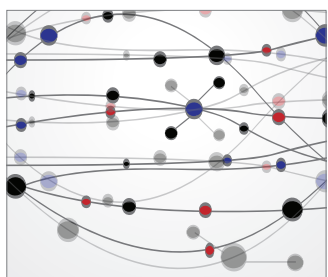

The Scientific World Journal
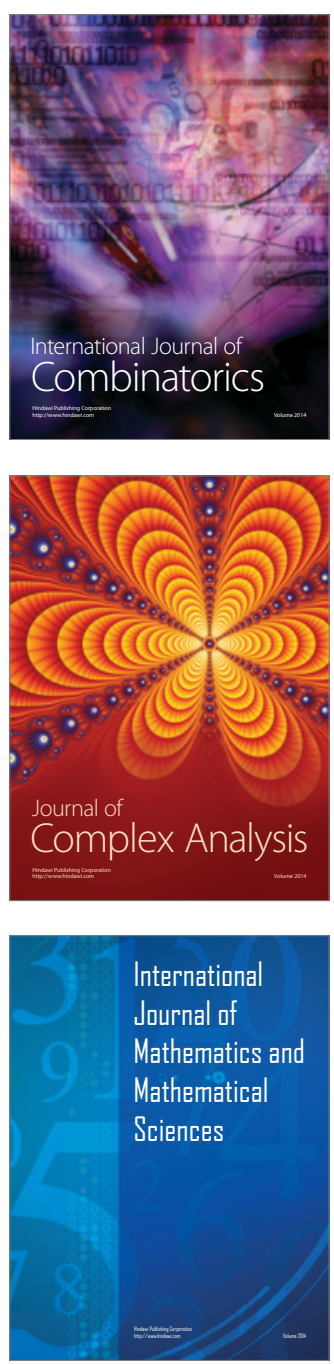
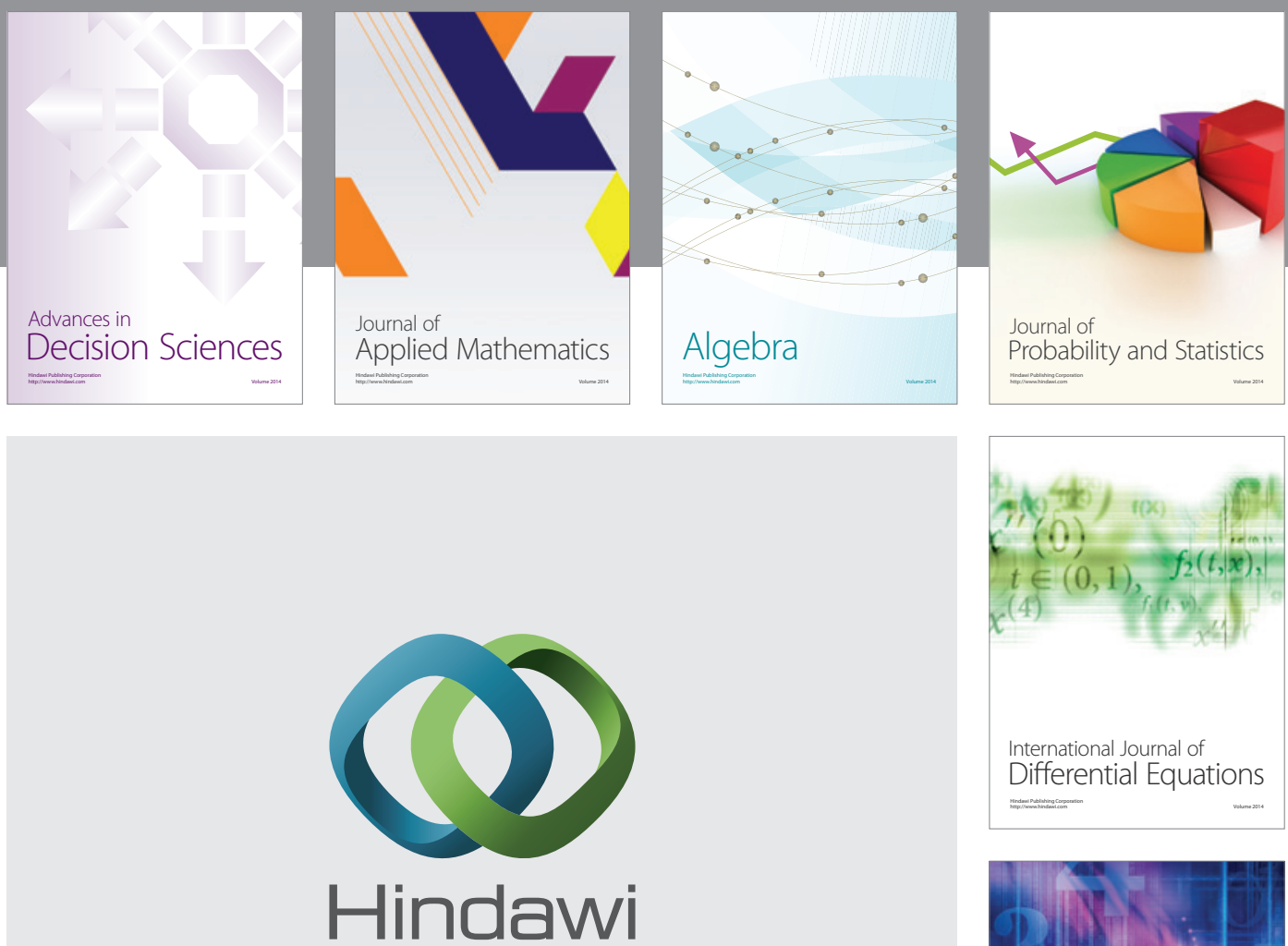

Submit your manuscripts at http://www.hindawi.com
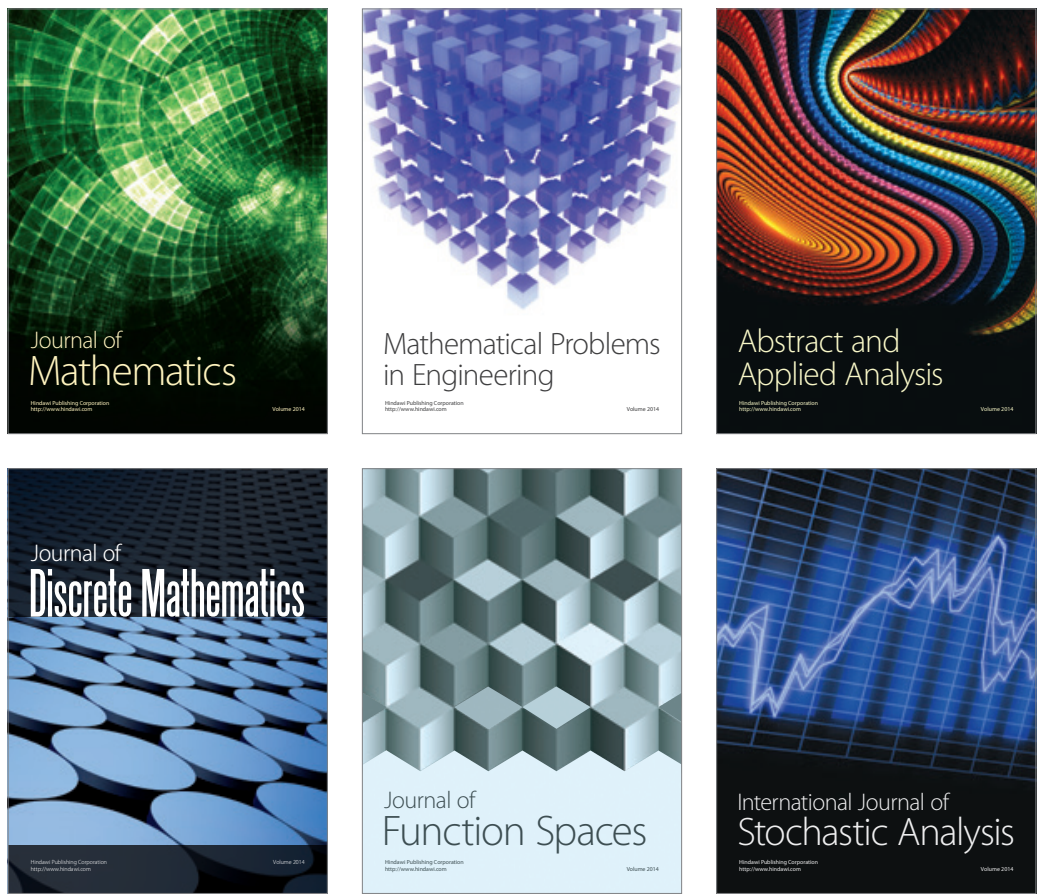

Journal of

Function Spaces

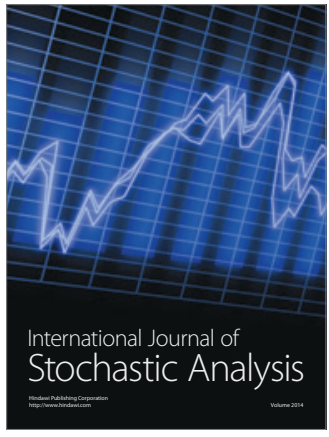

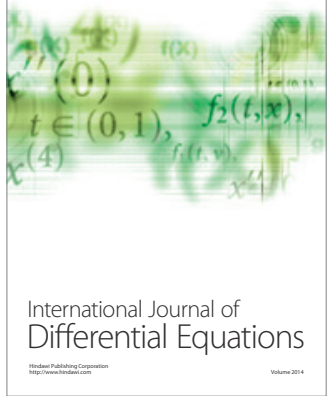
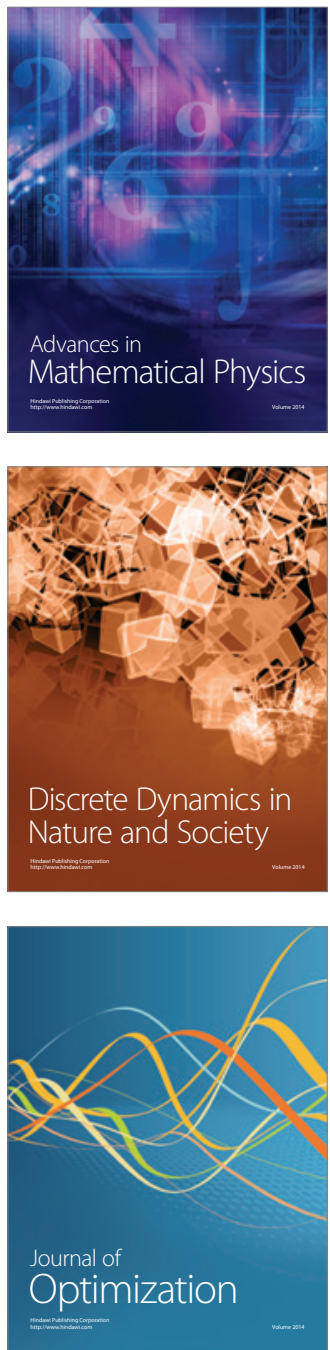